\title{
FACTORIZATION OF MATRICES AND MINKOWSKI'S CONJECTURE
}

\author{
by A. M. MACBEATH
}

(Received 31 October, 1960)

1. Introduction. Let $R^{n}$ denote real Euclidean space of $n$ dimensions. If

$$
\mathbf{x}=\left(x_{1}, \ldots, x_{n}\right) \in R^{n},
$$

define $\Pi \mathbf{x}=\left|x_{1} x_{2} \ldots x_{n}\right|$ and (as usual), $|\mathbf{x}|=\left(x_{1}^{2}+\ldots+x_{n}^{2}\right)^{\frac{1}{2}}$, so that, by the inequality of arithmetic and geometric means,

$$
\frac{|\mathbf{x}|^{2}}{n} \geqq(\Pi \mathbf{x})^{2 / n} .
$$

Let $\Lambda_{0}$ be the integer lattice, consisting of those points in $R^{n}$ whose co-ordinates are integers. A non-singular $n \times n$ matrix $M$ will be called a Minkowski matrix, if, for any point $\mathrm{a} \in R^{n}$, there exists a point $\mathbf{x} \in \Lambda_{0}$ such that

$$
\Pi(M \mathbf{x}-\mathbf{a}) \leqq 2^{-n}|\operatorname{det} M| .
$$

It was shown by Minkowski that, when $n=2$, every non-singular matrix is a Minkowski matrix, and that, for general $n$, every rational non-singular matrix is a Minkowski matrix. Minkowski is also said to have conjectured that every non-singular matrix is a Minkowski matrix, whatever the value of $n$. For $n=3$, this was proved by Remak [5], and a much simpler proof was given later by Davenport [2]. For $n=4$, it was proved by Dyson [3], who used a method similar to that of Remak and Davenport, but required the methods of algebraic topology to deal with some of the complications which arise in the higher dimension. Since this method depends also on the reduction of quadratic forms, it is quite likely that it might fail for higher values of $n$ even if the topological difficulties could be overcome. Therefore, an alternative proof for $n=3$, due to Birch and Swinnerton-Dyer [1], is of some interest, though in this dimension it is more complicated than Davenport's proof. A good deal of their analysis applies to general $n$, and they showed that for all $n$ there is a neighbourhood of the unit matrix $I$ that consists entirely of Minkowski matrices.

In the present note, I show more generally that, if $M_{0}$ is any non-singular matrix with rational elements, there is a neighbourhood of $M_{0}$ consisting entirely of Minkowski matrices. This is done by showing that the family of Minkowski matrices includes all those which can be expressed as a product of four matrices of certain types. It remains an open question whether all non-singular matrices can be factorized in such a manner, even for $n=3$. For $n=2$, the factorization is possible with little difficulty, and is, essentially, the basis of the proof due to Heilbronn given in Hardy and Wright's book [4, p. 394].

2. Definitions. A matrix $U$ is called unimodular if all its elements are integers and its determinant is \pm 1 . Since $U$ maps $\Lambda_{0}$ onto itself, $M U$ is a Minkowski matrix if $M$ is. 
An orthogonal matrix is one whose transpose is its own inverse $\left(A^{\prime}=A^{-1}\right)$ and a skew matrix $\Sigma$ is one whose transpose is its own negative. If $\exp \Sigma$ is defined by the convergent power series

$$
I+\Sigma+\frac{1}{2 !} \Sigma^{2}+\ldots
$$

then $\exp (-\Sigma)=(\exp \Sigma)^{-1}$, so that, if $\Sigma$ is skew, $\exp \Sigma$ is orthogonal.

A matrix $T$ is called unit-triangular if $t_{i i}=1, t_{i j}=0(i>j)$. A matrix $\tau$ is called zerotriangular if $\tau_{i j}=0(i \geqq j)$. We shall also require diagonal matrices $D$ with $d_{i j}=0$ for $i \neq j$.

A matrix $M$ is called a $D O T U$-matrix if it can be expressed as a product

$$
M=D A T U,
$$

where $D$ is non-singular diagonal, $A$ is orthogonal, $T$ is unit-triangular and $U$ is unimodular. Our results are as follows.

THEOREM 1. Every DOTU-matrix is a Minkowski matrix.

THEOREM 2. If $D, A, T, U$ are fixed matrices of the appropriate families, $A=\left(a_{i j}\right)$, and $\operatorname{det}\left(a_{i j}^{2}\right) \neq 0$, then the matrix $M_{0}=D A T U$ is an inner point of the family of Minkowski matrices.

COROLlaRY. Every rational non-singular matrix $R$ is an inner point of the family of Minkowski matrices.

For, by the theory of elementary divisors, $R=D T U$, and Theorem 2 can be applied with $A$ replaced by the unit matrix $I$.

3. Proof of Theorem 1. Let $\mathbf{M}$ denote the set of Minkowski matrices.

LEMMA 1. If $M \in \mathbf{M}$ and $D$ is non-singular diagonal, then $D M \in \mathbf{M}$.

Proof. We have

$$
\Pi D \mathbf{u}=|\operatorname{det} D| \Pi \mathbf{u} \text {. }
$$

If $\mathbf{a} \in R^{n}$ (so that $D^{-1} \mathbf{a} \in R^{n}$ ), then since $M \in \mathbf{M}$, there exists $\mathrm{x} \in \Lambda_{0}$ such that

$$
\Pi\left(M \mathbf{x}-D^{-1} \mathbf{a}\right) \leqq 2^{-n}|\operatorname{det} M| \text {. }
$$

Applying (3) with $\mathbf{u}$ replaced by $M \mathbf{x}-D^{-1} \mathbf{a}$, we have

$$
\Pi(D M \mathbf{x}-\mathbf{a})=|\operatorname{det} D| \Pi\left(M \mathbf{x}-D^{-1} \mathbf{a}\right) \leqq 2^{-n}|\operatorname{det} D M| \text {. }
$$

This proves the lemma.

Because of Lemma 1 , and because (see $\S 2$ ) $M U \in \mathbf{M}$ if $M \in \mathbf{M}$ and $U$ is unimodular, we need now only show that $A T \in \mathbf{M}$ if $A$ is orthogonal and $T$ unit-triangular. Let the columns of $A$ be $\mathrm{e}_{1}, \ldots, \mathrm{e}_{n}$ and let

$$
T=\left[\begin{array}{ccccc}
1 & t_{12} & \cdots & t_{1 n-1} & t_{1 n} \\
0 & 1 & \cdots & t_{2 n-1} & t_{2 n} \\
\vdots & \vdots & & \vdots & \\
0 & 0 & \cdots & 1 & t_{n-1 n} \\
0 & 0 & \cdots & 0 & 1
\end{array}\right] .
$$


Let $\mathbf{a} \in R^{n}$. Since $\mathbf{e}_{1}, \ldots, \mathbf{e}_{n}$ form an orthonormal set, a must be expressible in the form $\mathbf{a}=\alpha_{1} \mathbf{e}_{1}+\ldots+\alpha_{n} \mathbf{e}_{n}$. If $\mathbf{x}=\left(x_{1}, \ldots, x_{n}\right)$, then

$$
\mathbf{a}-A T \mathbf{x}=\beta_{1} \mathbf{e}_{1}+\ldots+\beta_{n} \mathbf{e}_{n}
$$

and a simple calculation shows that

$$
\begin{array}{lr}
\beta_{n}= & \alpha_{n}-x_{n}, \\
\beta_{n-1}= & \alpha_{n-1}-x_{n-1}-t_{n-1 n} x_{n}, \\
\ldots \ldots \ldots \ldots \ldots \ldots \ldots \ldots \ldots \ldots \ldots \ldots \ldots \ldots \ldots \ldots \ldots \ldots \ldots \ldots \ldots \ldots \ldots \ldots \ldots \ldots \ldots \ldots \ldots & t_{1 n} x_{n} .
\end{array}
$$

Choose an integer $x_{n}$ so that $\left|\beta_{n}\right| \leqq \frac{1}{2}$, then $x_{n-1}$ so that $\left|\beta_{n-1}\right| \leqq \frac{1}{2}$, etc. Then $\mathrm{x} \in \Lambda_{0}$, and, since the $e_{i}$ form an orthonormal set,

$$
|\mathbf{a}-A T \mathbf{x}|^{2}=\beta_{1}^{2}+\ldots+\beta_{n}^{2} \leqq \frac{1}{4} .
$$

By (1), it follows that

$$
\Pi(\mathbf{a}-A T \mathbf{x}) \leqq 2^{-n}=2^{-n} \operatorname{det} A T .
$$

Thus $A T \in \mathbf{M}$ and Theorem 1 is proved.

4. Proof of Theorem 2. Let $\tau, \Sigma, \Delta$ be variable matrices, $\tau$ zero-triangular with elements $\tau_{i j}(i<j), \Sigma$ skew symmetric with elements $\sigma_{i j}(i>j)$, and $\Delta$ a diagonal matrix with diagonal elements $\delta_{1}, \ldots, \delta_{n}$. This makes a total of $n^{2}$ variables in all. Consider the variable matrix

$$
M=D(I+\Delta) A(\exp \Sigma)(I+\tau) T U .
$$

For small $\Delta$ and all $\tau, \Sigma$, the matrix $M$ is a $D O T U$-matrix, since $D(I+\Delta)$ is diagonal, $A$ exp $\Sigma$ is orthogonal and $(I+\tau) T$ is unit triangular. The equation (5) represents $n^{2}$ equations in $n^{2}$ variables $\tau, \Sigma, \Delta$, and $M_{0}$ is the value for $\tau=\Sigma=\Delta=0$. We shall show that the Jacobian of these equations is not zero at this point. It then follows from the implicit function theorem that, if $M$ is close enough to $M_{0}$, the equations (5) have a solution $(\tau, \Sigma, \Delta)$, and hence $M_{0}$ is an inner point of the family of $D O T U$-matrices. Since the Jacobian at the origin is derived from the linear terms of the power series for $M$ in terms of $\tau, \Sigma, \Delta$, we shall neglect terms of higher than first order.

Make a linear transformation in $M$-space by putting

$$
P=A^{-1} D^{-1}\left(M-M_{0}\right) U^{-1} T^{-1} \text {. }
$$

Since $A, D, U, T$ are non-singular, $M$ will describe a neighbourhood of $M_{0}$ if and only if $P$ describes a neighbourhood of zero. Thus it suffices to calculate the Jacobian $\partial P / \partial(\tau, \Sigma, \Delta)$. Now

$$
P=A^{-1} \Delta A+\Sigma+\tau+\text { higher terms. }
$$


Take the elements $p_{i j}$ of $P$ in the following order, using the fact that $A$ is orthogonal, so that $A^{-1}=\left(a_{k i}\right)$.

(i) $(i<j) \quad p_{i j}=\tau_{i j}-\sigma_{j i}+$ terms in $\Delta+$ higher terms.

(ii) $(i>j) \quad p_{i j}=\sigma_{i j}+$ terms in $\Delta+$ higher terms.

(iii) $(i=j) \quad p_{i i}=\quad \sum_{k i}^{2} \delta_{k} \quad+$ higher terms.

If we write out the Jacobian matrix in partitioned form, it is seen to be

$$
\begin{array}{llrl} 
& \tau & \Sigma & \Delta \\
i<j & I & -I & L \\
i>j & 0 & I & M \\
i=j & 0 & 0 & N
\end{array}
$$

where $N$ is the matrix $\left(a_{k i}^{2}\right)$.

Thus $\partial P / \partial(\tau, \Sigma, \Delta)=\operatorname{det} N \neq 0$ by hypothesis. Hence $M_{0}$ is an inner point of the family of DOTU-matrices, and so an inner point of $\mathbf{M}$, by Theorem 1 .

\section{REFERENCES}

1. B. J. Birch and H. P. F. Swinnerton-Dyer, On the inhomogeneous minimum of the product of $n$ linear forms, Mathematika 3 (1956), 25-39.

2. H. Davenport, A simple proof of Remak's theorem on the product of three linear forms, J. London Math. Soc. 14 (1938), 47-51.

3. F. J. Dyson, On the product of four non-homogeneous linear forms, Ann. of Math. (2) 49, (1948), 82-109.

4. G. H. Hardy and E. M. Wright, An introduction to the theory of numbers (Oxford, 1938).

5. R. Remak, Verallgemeinerung eines Minkowskischen Satzes II, Math. Z. 18 (1923), 173-200.

\section{QUEEN'S COLLEGE}

DUNDEE 